\title{
A Belgian survey on the diagnosis of asthma- COPD overlap syndrome
}

\author{
This article was published in the following Dove Press journal: \\ International Journal of COPD \\ 13 February 2017 \\ Number of times this article has been viewed
}

\author{
Didier Cataldo,' Jean-Louis \\ Corhay,' Eric Derom, ${ }^{2}$ \\ Renaud Louis,' Eric \\ Marchand, ${ }^{3,4}$ Alain Michils, ${ }^{5}$ \\ Vincent Ninane, ${ }^{6}$ Rudi \\ Peché, ${ }^{7}$ Charles Pilette, ${ }^{8}$ \\ Walter Vincken, ${ }^{9}$ Wim \\ Janssens ${ }^{10}$
}

'Department of Respiratory Diseases, CHU Liège, University of Liège, Liège, ${ }^{2}$ Department of Respiratory Medicine, Ghent University Hospital, Ghent, ${ }^{3}$ Department of Respiratory Medicine, CHU - UCL - Namur, Université catholique de Louvain, Yvoir, ${ }^{4}$ Molecular Physiology Research Unit (URPhyM)-NARILIS, Laboratory of General Physiology, University of Namur, Namur, ${ }^{5}$ Chest Department, Erasme University Hospital, Université Libre de Bruxelles, Brussels, ${ }^{6}$ Department of Respiratory Medicine, University Hospital Saint-Pierre, Université Libre de Bruxelles, Brussels, ${ }^{7}$ Department of Respiratory Medicine, University Hospital Vésale, Montigny-le-Tilleul, ${ }^{8}$ Department of Respiratory Medicine, Cliniques universitaires St Luc, Université Catholique de Louvain, Brussels, ${ }^{9}$ Respiratory Division, University Hospital Brussels (UZ Brussel), Vrije Universiteit Brussel, Brussels, ${ }^{10}$ Department of Respiratory Diseases, University Hospitals Leuven, Leuven, Belgium

Correspondence: Didier Cataldo Department of Respiratory Diseases, University of Liège, CHU Sart-Tilman, Liège 4000, Belgium

Tel +3243662521

Fax +32 43662936

Email didier.cataldo@ulg.ac.be
Introduction: Patients with chronic airway disease may present features of both asthma and COPD, commonly referred to as asthma-COPD overlap syndrome (ACOS). Recommendations on their diagnosis are diffuse and inconsistent. This survey aimed to identify consensus on criteria for diagnosing ACOS.

Methods: A Belgian expert panel developed a survey on ACOS diagnosis, which was completed by 87 pulmonologists. Answers chosen by $\geq 70 \%$ of survey respondents were considered as useful criteria for ACOS diagnosis. The two most frequently selected answers were considered as major criteria, others as minor criteria. The expert panel proposed a minimal requirement of two major criteria and one minor criterion for ACOS diagnosis. Respondents were also asked which criteria are important for considering inhaled corticosteroids prescription in a COPD patient.

Results: To diagnose ACOS in COPD patients, major criteria were "high degree of variability in airway obstruction over time (change in forced expiratory volume in 1 second $\geq 400 \mathrm{~mL}$ )" and "high degree of response to bronchodilators ( $>200 \mathrm{~mL}$ and $\geq 12 \%$ predicted above baseline)". Minor criteria were "personal/family history of atopy and/or IgE sensitivity to $\geq 1$ airborne allergen", "elevated blood/sputum eosinophil levels and/or increased fractional exhaled nitric oxide", "diagnosis of asthma $<40$ years of age"; "symptom variability", and "age (in favor of asthma)". To diagnose ACOS in asthma patients, major criteria were "persistence of airflow obstruction over time (forced expiratory volume in 1 second/forced vital capacity ratio $<0.7$ )" and "exposure to noxious particles/gases, with $\geq 10$ pack-years for (ex-)smokers"; minor criteria were "lack of response on acute bronchodilator test"; "reduced diffusion capacity"; "limited variability in airway obstruction"; "age $>40$ years"; "emphysema on chest computed tomography scan".

Conclusion: Specific criteria were identified that may guide physicians to a more uniform diagnostic approach for ACOS in COPD or asthma patients. These criteria are largely similar to those used to prescribe inhaled corticosteroids in COPD.

Keywords: ACOS, airway obstruction, asthma, COPD, diagnosis, inhaled corticosteroids

\section{Introduction}

COPD and asthma are the two main chronic inflammatory diseases of the airways, and both are characterized by airflow limitation. While asthma is a clinically defined syndrome that typically develops early in life in association with allergy, COPD is generally not diagnosed below the age of 40 years and is caused by chronic exposure to noxious particles or gases, mainly tobacco smoke. COPD is characterized by a persistent airflow obstruction, defined by a ratio of forced expiratory volume in 1 second over forced vital capacity $\left(\mathrm{FEV}_{1} / \mathrm{FVC}\right.$ ratio) below 0.7 , or below the lower normal limit. ${ }^{1}$

In some patients, features of both diseases may coexist. They are commonly referred to as having asthma-COPD overlap syndrome (ACOS), as proposed by the 
Global Initiative for Asthma/Global Initiative for Chronic Obstructive Lung Disease (GINA/GOLD) statement and other working groups. ${ }^{2,3}$ The reported frequency of ACOS in populations of COPD patients varies widely, depending on the methodology of the study. ${ }^{4}$ ACOS prevalence rates range from $15 \%$ to $60 \%$, reflecting variations according to the age group, the population sampled, and the definitions of asthma and COPD. ${ }^{5}$ Based on observations of clinical studies, which tend to be more reliable, between $5 \%$ and $21 \%$ of COPD patients were diagnosed as having $\mathrm{ACOS}^{4}$ when using the Spanish criteria for diagnosis. ${ }^{2}$ The prevalence of ACOS in asthma patients with a smoking history was estimated to be $27 \%$ in a cross-sectional study in Finland. ${ }^{6}$ The disease presentation and response to therapy in ACOS patients might differ from those with COPD or asthma alone. Scientific data characterizing ACOS patients are scarce since these patients have generally been carefully excluded from clinical studies, which only include patients with an unequivocal diagnosis of asthma or COPD. ${ }^{7-9}$

ACOS patients are usually older than 40 years, but respiratory symptoms might have started in childhood or early adulthood. In most cases, there is a previous diagnosis of asthma by a health care professional, family history of asthma or allergies, or exposure to noxious gases or particulate matter. ${ }^{1}$

In ACOS patients, airflow limitation measured by spirometry is not fully reversible to $\beta_{2}$-mimetics, which is a major COPD feature, and chest X-ray findings are not specific. However, it is now well established that at least $39 \%$ of COPD patients actually display significant reversibility in response to bronchodilators, although the clinical meaning of this trait remains unclear. ${ }^{10}$

Airway inflammation measured in sputum samples or bronchoalveolar lavage is characterized by the presence of eosinophils and/or neutrophils. Interestingly, a cohort study conducted in Belgium recently showed that $37 \%$ of stable smoking COPD patients display sputum eosinophilia $\geq 3 \%,{ }^{11}$ further illustrating difficulties in categorizing such patients presenting with combined asthma and COPD features.

Repeated exacerbations decrease the quality of life and increase mortality and health-related costs in asthma and COPD patients. Medications such as inhaled corticosteroids (ICS), long-acting $\beta$-agonists (LABA), long-acting muscarinic antagonists (LAMA), or combinations of these medications were shown to have a favorable impact on both asthma and COPD exacerbations in placebo-controlled studies. ${ }^{12-19}$ A recent study demonstrated that LABA/LAMA associations might prevent exacerbations to a similar extent as compared to some LABA/ICS combinations. ${ }^{20}$ As COPD patients might mostly benefit from bronchodilator therapies (LAMA, LABA, or their combination), there is a challenge for the clinician to recognize an asthma component that will require ICS prescription. ${ }^{2}$

Given the lack of scientific evidence regarding the diagnosis and treatment of ACOS patients and the complexity of this phenotype, ${ }^{21}$ there is an important need for recommendations on its diagnosis, for both general practitioners and specialist pulmonologists. In addition, as recently proposed by $\mathrm{Sin}$ et $\mathrm{al}^{9}$ and others, a general and workable consensus on inclusion and exclusion criteria is urgently needed to conduct future studies on the diagnosis, management, and outcome of ACOS patients.

A national "ACOS working group" was set up in Belgium to reach consensus on criteria considered to be crucial for diagnosing ACOS. A diagnosis of asthma or COPD is often proposed after a first visit, while ACOS is rarely diagnosed at this time because longitudinal follow-up is needed. Therefore, criteria were assessed that could be considered for diagnosing ACOS either in an asthma patient or in a COPD patient. Additionally, because ACOS patients often require ICS therapy, criteria considered by specialists for initiating ICS therapy in COPD patients were also investigated.

\section{Methodology}

An online survey was set up by a panel of Belgian experts in the field of asthma and COPD. The results of this survey were analyzed and discussed by the expert panel.

\section{Inclusion/exclusion criteria for survey participation}

Pulmonologists from the three Belgian regions (BrusselsCapital, Flanders, and Wallonia) were identified through an available listing as potential participants to the survey if they were involved in the treatment of both adult asthma and COPD patients. Selected participants were contacted by e-mail or phone. Experts who prepared the survey questionnaire were excluded from participating.

\section{Survey}

The survey was developed by a panel of Belgian experts in the field of COPD and asthma from the three Belgian regions. The survey included four different questions. Predefined criteria to diagnose COPD and asthma patients as ACOS patients were identified by the experts based on 
published guidelines. ${ }^{2}$ This survey contained only general questions to pulmonologists about what they considered to be important for the diagnosis of a disease (ACOS); no patients were involved and no specific patient data were collected. Therefore, the expert panel declared that no involvement from an ethical committee was required, and this was confirmed by contacting the ethical committee from University of Liege (Belgium).

The first question of the survey was an open-ended question: "What are the most important criteria that make you think of ACOS in a patient? List at least three criteria and rank them from most to least important." The second and third questions were close-ended questions, in which the survey participants were asked to score predefined criteria by their level of importance, concerning the following topics: "What features are decisive to qualify a COPD patient as ACOS patient?" and "What features are decisive to qualify an asthma patient as ACOS patient?" Survey participants had no access to these criteria before having finalized their answer to question one. As a subquestion for questions two and three, the participants were asked to select the three most important criteria. The fourth question was again open: "What are the most important criteria for you to prescribe ICS to a COPD patient? List at least three criteria and rank them from most to least important."

To avoid bias in the scoring of the predefined criteria, questionnaires were designed to contain one criterion per screen-page, and correction of entries after completion of each page was not possible. In addition, two versions of the questionnaire were developed with differences in the order of questions two and three, in both main national languages (French and Dutch). Pulmonologists who accepted to participate were randomized to receive either one of the two available questionnaires. Duplication of questionnaires was avoided by asking each participant to encode his/her name and e-mail address.

\section{Analysis of the survey answers}

Answers to open-ended questions one and four were grouped in clusters according to similarity of provided answers. For example, for question one, the answers "smoking cessation", "history of smoking", "nicotine use", or "number of pack-years" were grouped together in the cluster "smoking".

For the close-ended questions two and three, a Likert scale ${ }^{22}$ was used with a score ranging from 1 to 7 . Scores were defined in the questionnaire as follows: $1=$ very irrelevant;
$2=$ irrelevant; $3=$ partially irrelevant; $4=I$ do not know $/$ not sure/no opinion; $5=$ partially relevant; $6=$ relevant; and $7=$ very relevant. During the analysis, scores $5-7$ were considered as "relevant" criteria and grouped together.

In addition to the answers on the four abovementioned questions, the following background information was collected for each survey participant: the type of hospital the pulmonologist is working at (nonacademic/academic), the number of years of practice $(0-5 / 5-10 / 10-20 />20$ years $)$, geographical location (Flanders, Wallonia, BrusselsCapital), repartition of asthma to COPD patients being treated by the pulmonologist $(0 \%-100 \%, 25 \%-75 \%$, $50 \%-50 \%, 75 \%-25 \%$, or $100 \%-0 \%$ ), and the sex of the pulmonologist.

\section{Guidance for the diagnosis of ACOS}

Based on the results from the survey, guidance for the diagnosis of ACOS was proposed by the expert panel. A methodology similar to the Spanish consensus on $\mathrm{ACOS}^{3}$ was implemented. Criteria that were considered relevant (Likert score 5-7) by $\geq 70 \%$ of the survey participants were retained for the development of guidance to consider ACOS in COPD or asthma patients.

The two criteria that were rated "relevant" by the highest percentage of pulmonologists for each close-ended question were chosen as major criteria, and all other criteria that surpassed the $70 \%$ cutoff for "relevant" criteria were included in the recommendations as minor criteria. The expert panel agreed that presence of two major criteria and at least one minor criterion was required for a reliable diagnosis of ACOS both in asthma and COPD patients.

\section{Statistics}

The results from the survey were analyzed using descriptive statistics (frequencies and percentages for categorical variables; mean, standard deviation, median, and first and third quartiles for continuous variables). Cross-tabulation was used for comparisons between categories. Percentages were calculated according to the number of available answers.

\section{Results \\ Survey participants}

The survey was conducted between October 21 and December 10,2015. Out of the 372 pulmonologists invited to participate in the survey, 87 adequately completed the questionnaire (response rate of 23.4\%) (Figure 1). Characteristics 


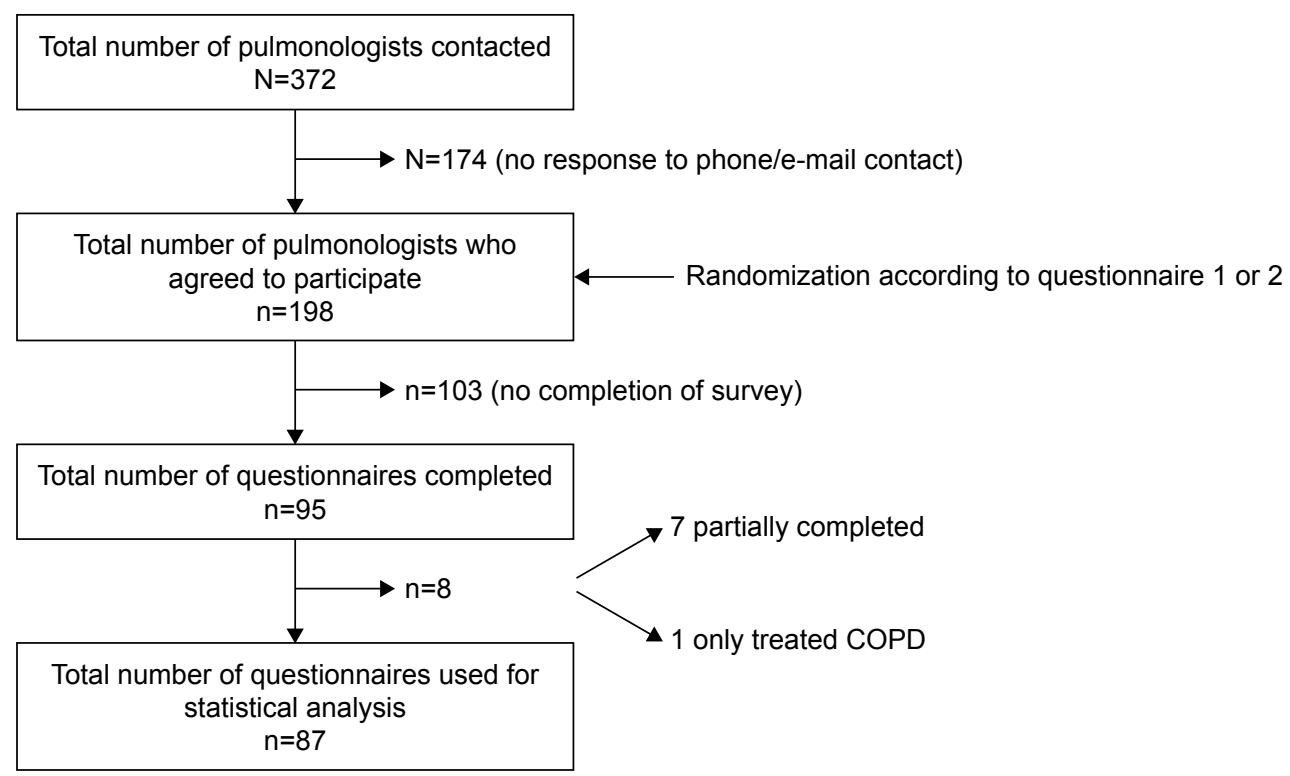

Figure I Participant flow chart.

of the participants are shown in Table 1. The participants can be considered as a representative sample of the whole Belgian population of pulmonologists invited to participate (data not shown).

\section{Important criteria for the diagnosis of ACOS}

As shown in Figure 2, 77\% of the pulmonologists mentioned "reversibility in lung function and/or airway obstruction" as the most important criterion related to the diagnosis of ACOS

Table I Characteristics of participants $(\mathrm{N}=87)$

\begin{tabular}{ll}
\hline Characteristics & $\mathbf{n}(\%)$ \\
\hline Sex & \\
Male & $49(56.3)$ \\
Female & $38(43.7)$ \\
Years of practice & \\
$>20$ & $35(40.2)$ \\
$10-20$ & $21(24.1)$ \\
$5-10$ & $19(21.8)$ \\
$<5$ & $12(13.8)$ \\
Hospital type & \\
Nonacademic & $66(75.9)$ \\
Academic & $21(24.1)$ \\
Region & \\
Flanders & $44(50.6)$ \\
Wallonia & $31(35.6)$ \\
Brussels & $12(13.8)$ \\
Proportion asthma/COPD treated & \\
$50 / 50$ & $41(47.1)$ \\
$25 / 75$ & $40(46.0)$ \\
$75 / 25$ & $6(6.9)$ \\
\hline
\end{tabular}

(survey open-ended question one). Other commonly reported criteria were "history or diagnosis of asthma", "allergy or atopy", and "smoking", which were very often cited in association with the most frequently mentioned criterion reversibility - $(60 \%-84 \%$ depending on the criterion $)$. All reported criteria are summarized in Table S1.

\section{Criteria to qualify a COPD patient as ACOS patient}

Fifteen criteria were predefined by the group of experts and ranked by each participant on a Likert scale (Figure 3, Table 2). The two criteria that were considered "relevant" (Likert score 5-7) by the highest percentage of pulmonologists and retained as major criteria were "degree of variability of airway obstruction" and "degree of response to bronchodilators". Other criteria that were considered to be relevant by more than $70 \%$ of the pulmonologists and proposed as minor criteria were "personal history of allergy or sensitivity to one or more allergens", "elevated eosinophils in sputum or blood or high nitric oxide levels", "diagnosis of asthma before the age of 40", "symptoms variability", and "age (in favor of asthma)".

Additionally, the participants were asked to select the three most important criteria to qualify a COPD patient as an ACOS patient. The most important criteria, as chosen by $49 \%$ of the pulmonologists, were "degree of response to bronchodilators" and "degree of variability in airway obstruction"; "asthma diagnosis before 40 years of age" was selected by $46 \%$; "personal or family history of atopy" 


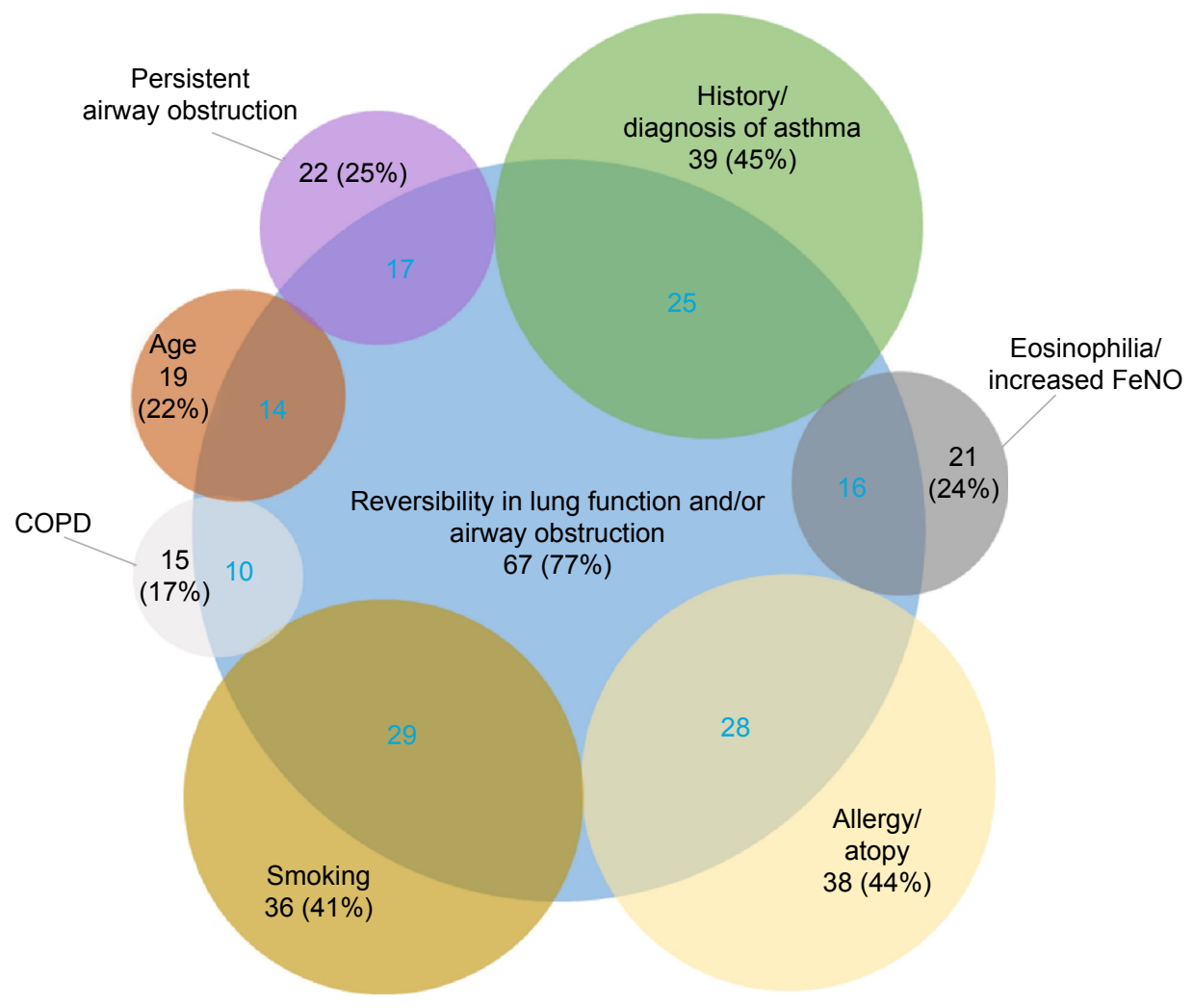

Figure 2 Major criteria for diagnosing ACOS.

Notes: The bubble size and presented number and percentage (in gray) indicate the number and percentage of pulmonologists who considered the criterion relevant for the diagnosis of ACOS. Overlap with the main reported criterion shows the number of each combination (indicated in blue) of both answers provided by pulmonologists. Overlap between the other criteria is not shown.

Abbreviations: ACOS, asthma-COPD overlap syndrome; FeNO, fractional exhaled nitric oxide.

\section{$\%$ pulmonologists who considered criterion relevant $(\mathrm{N}=87)$}

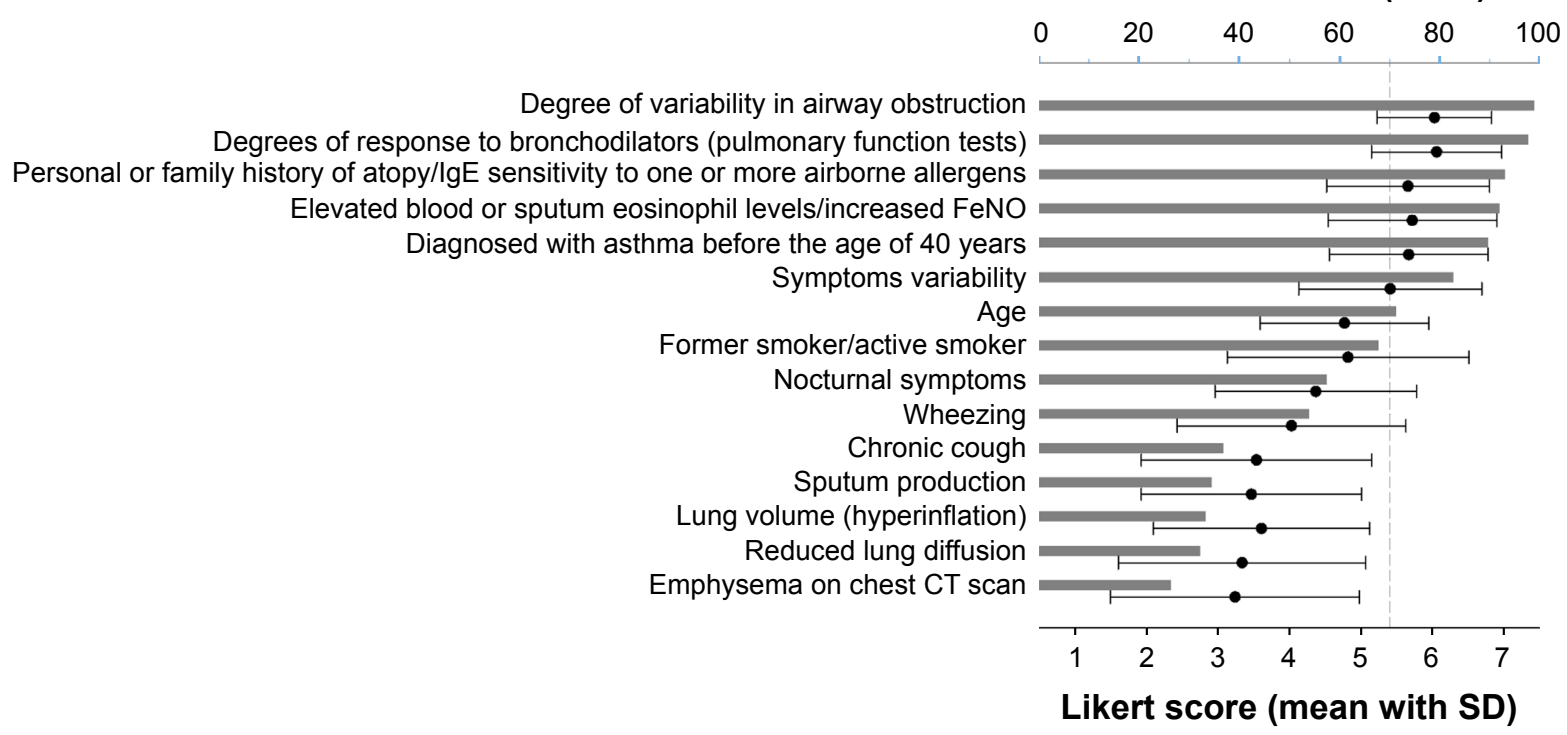

Figure 3 Features to diagnose a COPD patient as ACOS patient.

Notes: Gray bars show the percentage of pulmonologists who considered the criterion as "relevant" (Likert score 5-7). The two criteria considered relevant by most pulmonologists were retained as major criteria. Other criteria surpassing the $70 \%$ cutoff mark for relevancy (vertical dashed line) were considered as minor criteria. Black bullet shows mean Likert score (with SD).

Abbreviations: ACOS, asthma-COPD overlap syndrome; lgE, immunoglobulin E; FeNO, fractional exhaled nitric oxide; CT, computed tomography; N, number of pulmonologists; SD, standard deviation. 
Table 2 Criteria for ACOS diagnosis: guidance from the Belgian survey

\begin{tabular}{|c|c|}
\hline ACOS in a COPD patient & ACOS in an asthma patient \\
\hline Major criteria & Major criteria \\
\hline $\begin{array}{l}\text { High degree of variability in airway obstruction over time } \\
\text { (PFTs): FEV, variation } \geq 400 \mathrm{~mL}\end{array}$ & $\begin{array}{l}\text { Persistence over time of airflow obstruction (persistence of } \mathrm{FEV} / \mathrm{FVC} \\
\text { ratio }<0.7 \text { or }<\text { lower normal limit) }\end{array}$ \\
\hline $\begin{array}{l}\text { High degree of response to bronchodilators (PFTs): }>200 \mathrm{~mL} \\
\text { and } 12 \% \text { predicted above baseline }\end{array}$ & $\begin{array}{l}\text { Exposure to noxious particles or gases, with } \geq 10 \text { pack-years in case } \\
\text { of smoking for (ex-)smokers }\end{array}$ \\
\hline Minor criteria & Minor criteria \\
\hline $\begin{array}{l}\text { Personal or family history of atopy and/or IgE sensitivity to one } \\
\text { or more airborne allergens }\end{array}$ & Lack of response on acute bronchodilator test \\
\hline Elevated blood or sputum eosinophils or increased FeNO & Reduced lung diffusion capacity (on PFTs) \\
\hline Diagnosed with asthma before the age of 40 & Little variability in airway obstruction (PFTs) \\
\hline Symptom variability & Age in favor of COPD (ie, $>40$ years) \\
\hline Age (in favor of asthma) & Presence of emphysema on chest CT scan \\
\hline
\end{tabular}

Note: A diagnosis of ACOS is accepted in both COPD and asthma patients when the two major criteria and at least one minor criterion are met.

Abbreviations: ACOS, asthma-COPD overlap syndrome; FEV , forced expiratory volume in I second; FVC, forced vital capacity; PFTs, pulmonary function tests; IgE, immunoglobulin E; FeNO, fractional exhaled nitric oxide; CT, computed tomography.

by $45 \%$; and "elevated blood or sputum eosinophil levels or increased fractional exhaled nitric oxide (FeNO)" by $44 \%$ of participants (Table S2).

\section{Criteria to qualify an asthma patient as ACOS patient}

Fifteen criteria predefined by the group of experts were ranked by each participant on a Likert-scale (Figure 4, Table 2). As done for close-ended question two, the two criteria that were considered "relevant" (Likert score 5-7) by most pulmonologists were retained as major criteria. These were "persistence over time of an obstructive disorder (no normalization of $\mathrm{FEV}_{1} / \mathrm{FVC}$ ratio)" and "smoker (former or active smoker)". Other criteria that were considered to be relevant by more than $70 \%$ of the pulmonologists were indicated as minor criteria. These were "degree of response to bronchodilators, as measured on pulmonary function tests (PFTs)", "reduced lung diffusion capacity", "degree of variability in airway obstruction on PFTs", "age", and "presence of emphysema on chest CT scan".

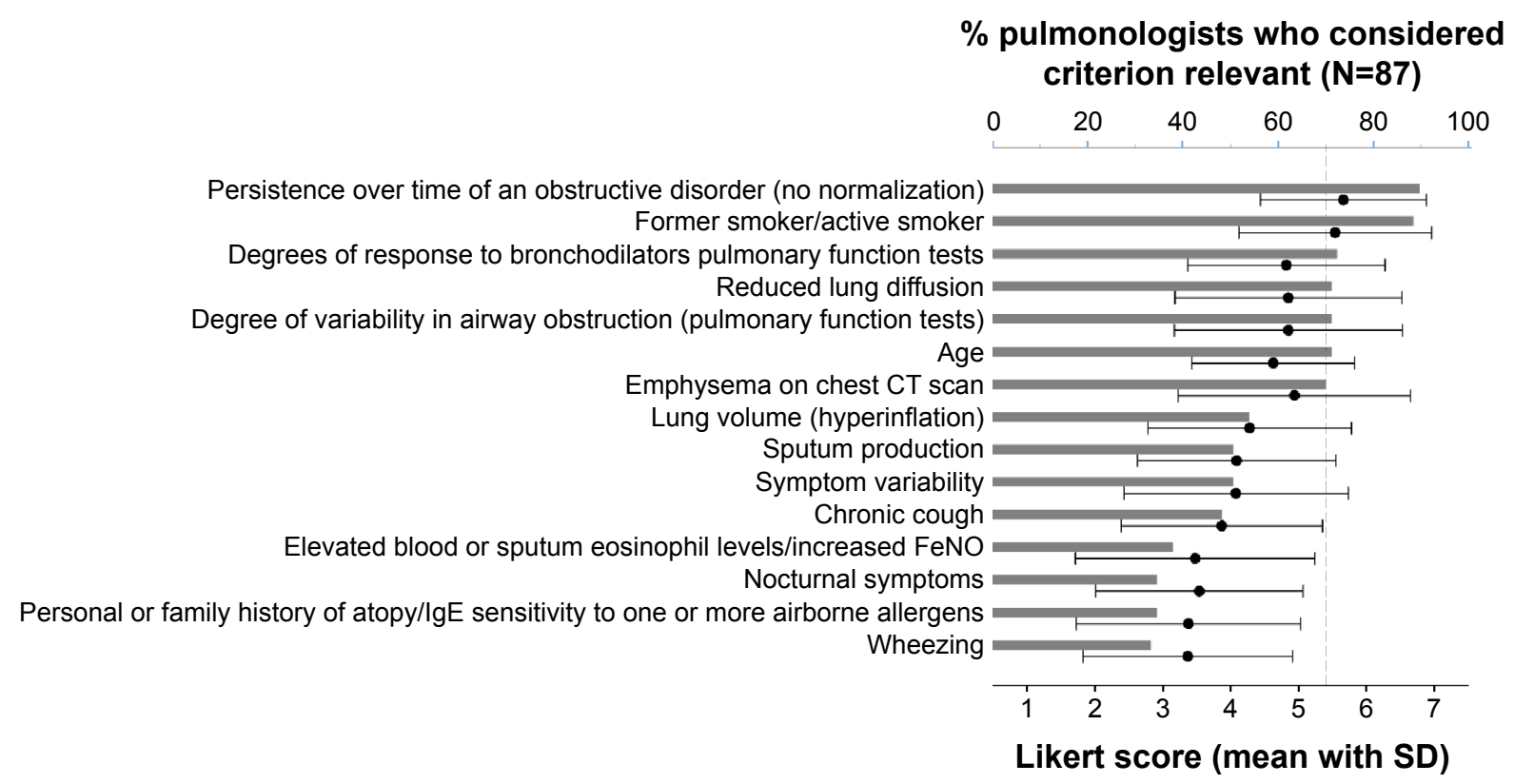

Figure 4 Features to diagnose an asthma patient as ACOS patient.

Notes: Figure shows the percentage of pulmonologists who considered the criterion as "relevant" (Likert score 5-7). The two criteria considered relevant by most pulmonologists were retained as major criteria. Other criteria surpassing the $70 \%$ cutoff mark for relevancy (vertical dashed line) were considered as minor criteria. Black bullet shows mean Likert score (with SD).

Abbreviations: ACOS, asthma-COPD overlap syndrome; CT, computed tomography; FeNO, fractional exhaled nitric oxide; IgE, immunoglobulin E; N, number of pulmonologists; SD, standard deviation. 
Similar results were obtained when the pulmonologists were asked to select the three most important criteria. "Persistence over time of an obstructive disorder" was selected by $70 \%$ of pulmonologists, "smoking (former or active smoker)" by $66 \%$, "presence of emphysema on chest computed tomography (CT) scan" by $45 \%$, and "reduced lung diffusion capacity" by $32 \%$ (Table S3).

\section{Criteria to prescribe ICS to a COPD patient}

When the pulmonologists were asked to state the most important criteria to prescribe ICS to a COPD patient, "exacerbations" was the most frequently mentioned criterion, reported by $89 \%$ of survey participants. Other commonly reported criteria were "eosinophilia/increased FeNO" and "reversibility in lung function and/or airway obstruction" (Figure 5).

\section{Guidance for ACOS diagnosis proposed by the expert panel}

It was agreed upon by the expert panel that presence of two major criteria and at least one minor criterion would be required for the diagnosis of ACOS, both in asthma and COPD patients. The criteria that were proposed based on the findings from the survey are summarized in Table 2. Where possible, the findings from the survey were expanded with cutoff values.

\section{Discussion}

This survey documents the criteria considered as relevant by pulmonologists in Belgium to diagnose ACOS in patients suffering from asthma or COPD, and accordingly proposes a guideline for ACOS diagnosis is.

While patients with characteristics of both asthma and COPD have been largely excluded from clinical trials, ACOS has increasingly retained interest. In 2014, guidelines for the diagnosis of ACOS were proposed in a joint effort of GINA and GOLD, ${ }^{2}$ and the syndrome is also appearing in national clinical practice guidelines. ${ }^{23-27}$

Clearly defined criteria for the diagnosis of ACOS are important for several reasons. First, ACOS patients probably display specific clinical and functional trajectories with a generally more severe disease and worse prognosis than asthma or COPD patients without overlap. For example, ACOS patients have a higher frequency of exacerbations and subsequent hospitalizations, which result in significantly higher health care costs compared to patients with COPD or asthma alone. ${ }^{28-32}$ Second, there are also indications that ACOS patients display a systemic disease with inflammation ${ }^{33,34}$ and may even have an increased risk for the development of nonrespiratory cancers. ${ }^{35}$ Finally, the societal burden impacting daily activities is believed to be more important in ACOS patients than in patients with asthma or COPD alone. ${ }^{36}$

In the experience of the experts, ACOS will rarely appear as a first clinical diagnosis; physicians usually start with the most likely diagnosis (asthma or COPD), and may then move to a diagnosis of ACOS during follow-up based on the evolution across time (eg, lung function, variability in symptoms) of the patient. Therefore, the two close-ended questions of this survey were set up to diagnose ACOS either in a COPD or in an asthma patient.

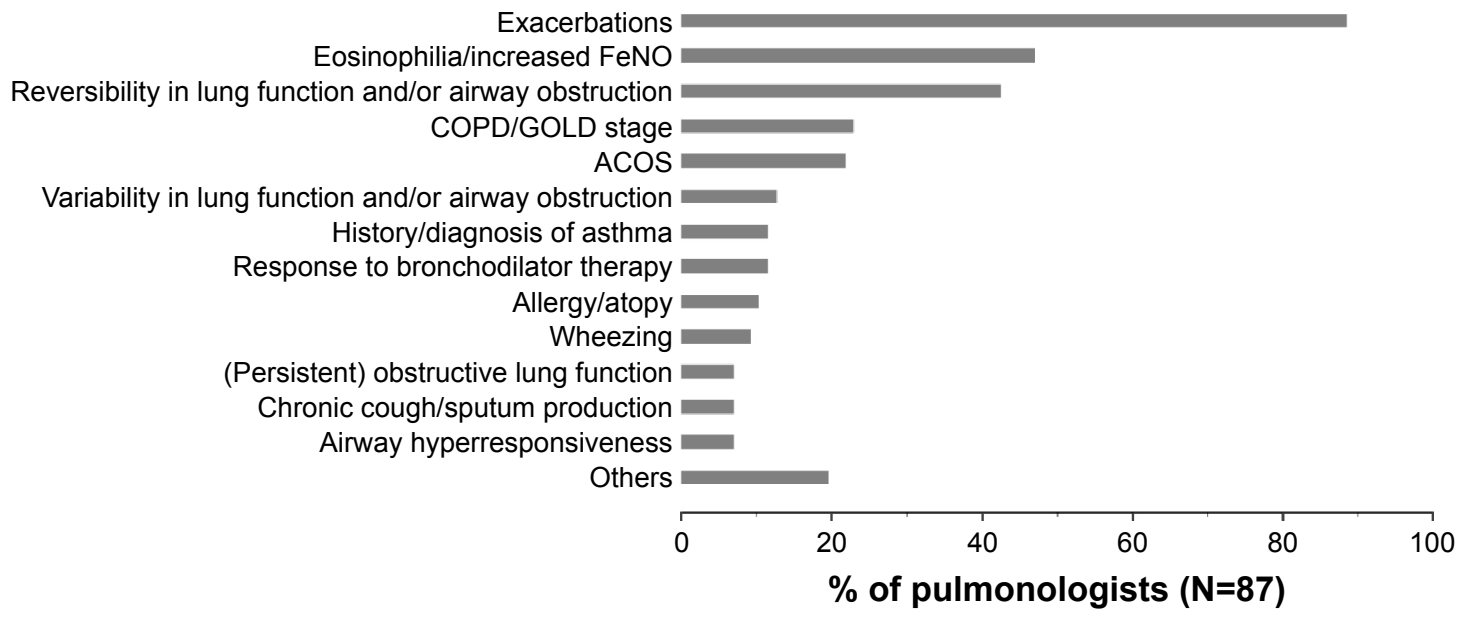

Figure 5 Major criteria for prescribing ICS to COPD patients.

Note: Figure shows the percentage of pulmonologists who considered the criterion important for prescribing ICS to COPD patients.

Abbreviations: ICS, inhaled corticosteroids; FeNO, fractional exhaled nitric oxide; GOLD, Global Initiative for Chronic Obstructive Lung Disease; ACOS, asthma-COPD overlap syndrome; $\mathrm{N}$, number of pulmonologists. 


\section{Criteria to diagnose ACOS in COPD or asthma patients}

About $80 \%$ of participating pulmonologists considered "degree of reversibility in lung function and/or airway obstruction" as an important criterion related to ACOS (regardless of the previous diagnosis of the patient, ie, COPD or asthma). Since other answers showed a lower level of consensus among pulmonologists (50\% or less similar answers), it was difficult to propose a set of clear-cut criteria based on the answers provided to open-ended question one. As already mentioned, ACOS is rarely diagnosed at the initial assessment, and so it is easier to develop recommendations considering a patient with a first presumed diagnosis of COPD or asthma. Of note, the level of consensus was higher for the ranking of predefined criteria for the diagnosis of ACOS in a COPD patient compared to an asthma patient.

Based on the answers of pulmonologists to the survey and the subsequent discussion by the expert panel, recommendations are proposed to diagnose ACOS in COPD and asthma patients (Table 2). In both COPD and asthma, the patient should meet the two major criteria and at least one minor criterion to be classified as a possible ACOS patient.

The two major criteria to diagnose a COPD patient as potential ACOS patient were "high degree of variability in airway obstruction over time" and "pronounced response to bronchodilators". The cutoffs proposed by the expert panel are an increase of $\geq 400 \mathrm{~mL}$ over time as degree of variability in airway obstruction, ${ }^{2}$ an increase in $\mathrm{FEV}_{1}$ of $>200 \mathrm{~mL}$, and a $12 \%$ increase relative to baseline level for acute response to bronchodilators. ${ }^{37}$

The two major criteria to diagnose an asthma patient as ACOS were "persistence over time of an obstructive disorder" and "smoker (former/active)". The panel of experts recommends to include "exposure to noxious particles and gases", also so as to encompass other exposures than smoking, for example professional exposures. In addition, for smoking, a cutoff of $\geq 10$ pack-years was defined, which is considered to reflect a significant exposure to tobacco smoke.

The other quantifiable minor criteria were not further detailed by the expert panel since scientific data are lacking to define validated cutoff values. These minor criteria were elevated eosinophils or increased FeNO and symptom variability for COPD patients, and lack of response on acute bronchodilator test, reduced lung diffusion capacity, little variability in airway obstruction, and presence of emphysema on chest CT scan for asthma patients. As there is no consensus in the literature, precise cutoff levels for eosinophil counts and FeNO levels were not proposed as part of the criteria. However, levels of 300 eosinophils $/ \mathrm{mm}^{3}$ have been suggested elsewhere; ${ }^{9,38}$ for FeNO levels, suggested cutoff values to classify patients as having ACOS range from $19 \mathrm{bbp}^{39}$ over $22.5 \mathrm{bbp}^{40}$ to $35 \mathrm{bbp.}^{41}$

The findings of this survey are in general agreement with criteria reported for diagnosis of ACOS in COPD patients in the Spanish consensus paper ${ }^{3}$ and with the criteria for ACOS diagnosis proposed by a global expert panel. ${ }^{9}$ Bronchodilator reversibility, history of asthma, and airway eosinophilia are widely accepted criteria to raise suspicion for an asthma component in a COPD patient. ${ }^{3,9}$ Bronchodilator response was indicated as a major criterion in this survey as well as by the Spanish and global expert panels, although the Belgian experts proposed a less stringent cutoff $(>200 \mathrm{~mL}$ and $12 \%$ above baseline, compared to $>400 \mathrm{~mL}$ and $\geq 15 \%$ in the other studies $\left.{ }^{3,9}\right)$. The other two major criteria from the Spanish consensus ${ }^{3}$ (history of asthma before age 40 , and eosinophilia) were indicated as minor criteria in the Belgian proposal. The global expert panel ${ }^{9}$ also chose history of asthma before 40 years of age as a major criterion, while elevated blood eosinophils was a minor criterion. The other two major criteria proposed by the global expert panel ${ }^{9}$ (persistent airflow obstruction and air pollution exposure or $\geq 10$ pack-years) corresponded to the major criteria proposed by the Belgian expert panel for the diagnosis of ACOS in an asthma patient. Surprisingly, one major criterion in COPD patients proposed by the Belgian experts - high variability in airway obstruction over time - was not mentioned in the two other studies. The Belgian experts feel this criterion is important as it may comprise an unexpected major treatment response over time, which is indicative of major reversibility and a preferred asthma feature. Finally, the number of major and minor criteria that should be present to diagnose a patient with ACOS differs across the studies. As there is no gold standard, it is impossible to compare which of them is best.

The strengths and novelty of the Belgian expert recommendations are found in the fact that they provide, for the first time, specific criteria for previously diagnosed asthma patients in whom the suspicion of ACOS is raised during follow-up. The importance of diagnosing ACOS in asthma patients has been demonstrated in a recent assessment on the long-term prognosis of ACOS patients. ${ }^{42}$ While a poor prognosis was observed for all ACOS patients, the prognosis seemed to be affected by the age at diagnosis of asthma. Indeed, ACOS patients with late-onset asthma (after the age of 40) display the worst prognosis, indicating the need for early diagnosis and closer follow-up. ${ }^{42}$ Expert groups in Czech Republic and Finland included ACOS in their recently published national 
guidelines for the diagnosis and treatment of COPD. ${ }^{23,27}$ These two groups built their guidelines based on the GOLD report and the Spanish COPD guidelines, which are similar to the approach used here for the development of the survey.

Most importantly, evidence-based guidelines for the treatment of ACOS patients do not exist, and without a correct diagnosis, patients might not receive the appropriate treatment. To clearly delineate this important group of patients for future interventional studies, a consensus on whom to consider as an ACOS patient is required. The problem of inconsistent definitions used in treatment studies, which makes it difficult to determine the most effective therapy for a particular patient, has already been brought forward by other authors..$^{9,43}$ The present survey largely supports their suggestion of extensive phenotypic characterization of individual patients before inclusion in clinical trials. However, although Postma and Rabe ${ }^{43}$ found it currently premature to designate ACOS as a disease entity, the Belgian expert panel believes that the criteria proposed here and by other groups will provide valuable guidance for physicians in the assessment of their patients and will make possible the design of specific studies focused on these specific patients.

\section{Criteria considered by Belgian respiratory physicians for initiation of ICS in COPD patients}

In accordance with the GOLD statement and a number of previous studies showing that ICS reduce the risk of exacerbations in COPD patients with frequent exacerbations, ${ }^{44-46}$ about $90 \%$ of pulmonologists in this survey considered "exacerbations" as a criterion to prescribe ICS to a COPD patient. Nevertheless, while triple therapy (LABA/LAMA/ICS) is recommended only for COPD patients classified as GOLD D $\left(\mathrm{FEV}_{1}\right.$ after bronchodilator $<50 \%$ predicted and/or a history of two or more exacerbations per year; or one or more hospitalizations for a COPD exacerbation as well as a modified Medical Research Council [mMRC] grade $\geq 2$; or a COPD assessment test score $\geq 10),{ }^{47}$ it is very often also prescribed to COPD patients classified as GOLD A, B, or C. ${ }^{48}$ The appropriateness of such a triple therapy in non-GOLD D patients is questionable. The most common pathway to triple therapy was observed to be a prescription of LABA/ICS combination before COPD diagnosis, followed by addition of LAMA at the time of appropriate COPD diagnosis. ${ }^{48}$ The experts who conceived the present survey recommend reasonable use of (low-dose) ICS in patients with frequent bacterial exacerbations in view of the increased risk of community-acquired pneumonia and other respiratory infections.
In COPD patients, eosinophilic airway inflammation may play a role in exacerbations, especially in more severe cases. Sputum eosinophils have been identified as a risk factor for COPD exacerbations upon withdrawal of ICS in COPD. ${ }^{49,50}$ Thus, increased sputum eosinophil levels appear to be a useful indicator for initiating ICS therapy in order to reduce the risk of exacerbations.

In clinical practice, patients with a mixed phenotype often receive a combination of asthma and COPD medications; however, this is merely based on extrapolation. In the survey presented here, at least $22 \%$ of the participating pulmonologists indicated that a diagnosis of ACOS is a major criterion for initiating ICS in COPD patients. Other studies are definitely needed to provide the necessary evidence for validating this common practice. Such studies might validate a treatment approach based on a LABA/LAMA combination as standard treatment for COPD, an ICS/LABA combination as standard for asthma, and a triple therapy in case of ACOS.

\section{Survey strengths and limitations}

About a quarter of the pulmonologists who were invited to participate in the survey successfully completed the questionnaire. This was, to our knowledge, the largest group of specialist practitioners who were consulted to develop a consensus on the diagnosis of ACOS. Additionally, this paper is the first to provide guidance for the diagnosis of ACOS in asthma patients.

A limitation of the findings presented here is the fact that insufficient information is currently available to provide specific cutoffs for the minor criteria identified for the diagnosis of ACOS in asthma and COPD patients. The specialist practitioners should assess, based on their experience, if the specified criterion is met in their COPD or asthma patients.

\section{Conclusion}

Our survey points out the criteria considered by Belgian pulmonologists as relevant to the diagnosis of ACOS, and based on this survey, a consensus on ACOS diagnosis in patients suffering from asthma or COPD is proposed. Criteria used to classify COPD patients as ACOS were largely similar to those used to prescribe ICS in COPD patients in Belgium (apart from COPD exacerbations, an indication accepted for a long time).

\section{Acknowledgments}

The authors wish to thank all the pulmonologists who participated in this survey. The authors acknowledge the financial support of AstraZeneca. The authors also wish to thank XPE 
Pharma \& Science for the setup, conduct, and analysis of the survey, and Bram Blomme, Joke Vandewalle, and Wouter Houthoofd (XPE Pharma \& Science) for writing assistance and coordination of manuscript development. The setup, conduct, and analysis of the survey and the development of this manuscript were funded by AstraZeneca.

\section{Disclosure}

$\mathrm{DC}$ is the founder of Aquilon Pharmaceuticals, received speaker fees from AstraZeneca, Boehringer Ingelheim, Novartis, MundiPharma, Chiesi, and GSK and received consultancy fees from AstraZeneca, Boehringer Ingelheim, and Novartis for the participation on advisory boards. J-LC received speaker fees from AstraZeneca, Boehringer Ingelheim, Novartis, and GSK and received honoraria for the participation to advisory boards organized by AstraZeneca, Novartis, GSK, and Boehringer Ingelheim. ED reports having received travel grants from Boehringer Ingelheim, GSK, and AstraZeneca to attend international congresses; has participated in advisory boards by Boehringer Ingelheim, Chiesi, Cipla, and AstraZeneca, (for which a fee was given); and has received speaker's fees from Boehringer Ingelheim, GSK AstraZeneca, and Menarini to give scientific presentations to Belgian GP groupings (all not related to this work). His clinical department received financial support from Boehringer Ingelheim and Novartis to perform clinical studies. RL received research grants from AstraZeneca, Chiesi, GSK, and Novartis and received honoraria for advisory boards from AstraZeneca, Novartis, and Chiesi. EM received honoraria for the participation to advisory boards organized by AstraZeneca, Novartis, and Boehringer Ingelheim as well as speaker's fee from Novartis and Boehringer Ingelheim. AM has received speaker fees from AstraZeneca, Novartis, Chiesi, GSK, and Stallergènes and received consultancy fees from AstraZeneca, Chiesi, and Novartis for the participation on advisory boards. AM received research grants from AstraZeneca, Novartis, and Chiesi. VN is member of advisory boards of Boehringer Ingelheim, GSK, Novartis, and AstraZeneca and received speakers fees for lectures for these companies. RP is member of advisory board and task forces for AstraZeneca, Boehringer Ingelheim, Chiesi, GSK, and Novartis. CP received research grants from AstraZeneca, GSK, Novartis, Chiesi, and TEVA and has been a member of advisory boards for these companies and for MundiPharma. WV has received speaker fees or consultancy fees from AstraZeneca, Boehringer Ingelheim, Novartis, MundiPharma, Chiesi, and GSK. WJ is holder of the AZ Foundation KUL Chair in Respiratory Diseases. He received research grants from Boehringer Ingelheim, GSK, Novartis, and Chiesi. He is a member of advisory boards of Boehringer Ingelheim, GSK, Novartis, Chiesi, and AstraZeneca and received speakers fees for lectures for these companies. The authors report no other conflicts of interest in this work.

\section{References}

1. Aggarwal AN, Gupta D, Agarwal R, Jindal SK. Comparison of the lower confidence limit to the fixed-percentage method for assessing airway obstruction in routine clinical practice. Respir Care. 2011; 56(11):1778-1784.

2. Global Initiative for Asthma. Diagnosis of Diseases of Chronic Airflow Limitation: Asthma, COPD and Asthma-COPD Overlap Syndrome (ACOS). A joint project of GINA and GOLD. 2015.

3. Soler-Cataluna JJ, Cosio B, Izquierdo JL, et al. Consensus document on the overlap phenotype COPD-asthma in COPD. Arch Bronconeumol. 2012;48(9):331-337.

4. Barrecheguren M, Esquinas C, Miravitlles M. The asthma-chronic obstructive pulmonary disease overlap syndrome (ACOS): opportunities and challenges. Curr Opin Pulm Med. 2015;21(1):74-79.

5. Bateman ED, Reddel HK, van Zyl-Smit RN, Agusti A. The asthmaCOPD overlap syndrome: towards a revised taxonomy of chronic airways diseases? Lancet Respir Med. 2015;3(9):719-728.

6. Kiljander T, Helin T, Venho K, Jaakkola A, Lehtimaki L. Prevalence of asthma-COPD overlap syndrome among primary care asthmatics with a smoking history: a cross-sectional study. NPJ Prim Care Respir Med. 2015;25:15047.

7. Miravitlles M, Calle M, Soler-Cataluna JJ. Clinical phenotypes of COPD: identification, definition and implications for guidelines. Arch Bronconeumol. 2012;48(3):86-98.

8. Miravitlles M. Individually-tailored treatment of chronic obstructive pulmonary disease: a proposed change. Arch Bronconeumol. 2009;45(Suppl 5):27-34.

9. Sin DD, Miravitlles M, Mannino DM, et al. What is asthma-COPD overlap syndrome? Towards a consensus definition from a round table discussion. Eur Respir J. 2016;48(3):664-673.

10. Tashkin DP, Celli B, Decramer M, et al. Bronchodilator responsiveness in patients with COPD. Eur Respir J. 2008;31(4):742-750.

11. Schleich F, Corhay JL, Louis R. Blood eosinophil count to predict bronchial eosinophilic inflammation in COPD. Eur Respir J. 2016; 47(5):1562-1564.

12. Calverley P, Pauwels R, Vestbo J, et al. Combined salmeterol and fluticasone in the treatment of chronic obstructive pulmonary disease: a randomised controlled trial. Lancet. 2003;361(9356):449-456.

13. Calverley PM, Anderson JA, Celli B, et al. Salmeterol and fluticasone propionate and survival in chronic obstructive pulmonary disease. N Engl J Med. 2007;356(8):775-789.

14. Calverley PM, Boonsawat W, Cseke Z, Zhong N, Peterson S, Olsson H. Maintenance therapy with budesonide and formoterol in chronic obstructive pulmonary disease. Eur Respir J. 2003;22(6):912-919.

15. Pauwels RA, Lofdahl CG, Postma DS, et al. Effect of inhaled formoterol and budesonide on exacerbations of asthma. Formoterol and Corticosteroids Establishing Therapy (FACET) International Study Group. N Engl J Med. 1997;337(20):1405-1411.

16. Rabe KF, Atienza T, Magyar P, Larsson P, Jorup C, Lalloo UG. Effect of budesonide in combination with formoterol for reliever therapy in asthma exacerbations: a randomised controlled, double-blind study. Lancet. 2006;368(9537):744-753.

17. Szafranski W, Cukier A, Ramirez A, et al. Efficacy and safety of budesonide/formoterol in the management of chronic obstructive pulmonary disease. Eur Respir J. 2003;21(1):74-81.

18. Tashkin DP, Celli B, Senn S, et al. A 4-year trial of tiotropium in chronic obstructive pulmonary disease. $N$ Engl J Med. 2008;359(15): $1543-1554$. 
19. Welte T, Miravitlles M, Hernandez P, et al. Efficacy and tolerability of budesonide/formoterol added to tiotropium in patients with chronic obstructive pulmonary disease. Am J Respir Crit Care Med. 2009;180(8):741-750.

20. Wedzicha JA, Banerji D, Chapman KR, et al. Indacaterol-glycopyrronium versus salmeterol-fluticasone for COPD. N Engl J Med. 2016; 374(23):2222-2234.

21. Tho NV, Park HY, Nakano Y. Asthma-COPD overlap syndrome (ACOS): a diagnostic challenge. Respirology. 2016;21(3):410-418.

22. Likert R. A technique for the measurement of attitudes. Arch Psychol. 1932(140):1-55.

23. Kankaanranta H, Harju T, Kilpelainen M, et al. Diagnosis and pharmacotherapy of stable chronic obstructive pulmonary disease: the Finnish guidelines. Basic Clin Pharmacol Toxicol. 2015;116(4):291-307.

24. Miravitlles M, Soler-Cataluna JJ, Calle M, et al. A new approach to grading and treating COPD based on clinical phenotypes: summary of the Spanish COPD guidelines (GesEPOC). Prim Care Respir J. 2013;22(1):117-121.

25. Nagai A. [Guidelines for the diagnosis and management of chronic obstructive pulmonary disease: 3rd edition]. Nihon Rinsho. 2011;69(10): 1729-1734. Japanese.

26. O’Donnell DE, Aaron S, Bourbeau J, et al. Canadian Thoracic Society recommendations for management of chronic obstructive pulmonary disease - 2007 update. Can Respir J. 2007;14(Suppl B):5B-32B.

27. Koblizek V, Chlumsky J, Zindr V, et al. Chronic obstructive pulmonary disease: official diagnosis and treatment guidelines of the Czech Pneumological and Phthisiological Society; a novel phenotypic approach to COPD with patient-oriented care. Biomed Pap Med Fac Univ Palacky Olomouc Czech Repub. 2013;157(2):189-201.

28. Andersen H, Lampela P, Nevanlinna A, Saynajakangas O, Keistinen T. High hospital burden in overlap syndrome of asthma and COPD. Clin Respir J. 2013;7(4):342-346.

29. Brzostek D, Kokot M. Asthma-chronic obstructive pulmonary disease overlap syndrome in Poland. Findings of an epidemiological study. Postepy Dermatol Alergol. 2014;31(6):372-379.

30. Gerhardsson de Verdier M, Andersson M, Kern DM, Zhou S, Tunceli O. Asthma and chronic obstructive pulmonary disease overlap syndrome: doubled costs compared with patients with asthma alone. Value Health. 2015;18(6):759-766.

31. Hardin M, Silverman EK, Barr RG, et al. The clinical features of the overlap between COPD and asthma. Respir Res. 2011;12:127.

32. Menezes AM, Montes de Oca M, Perez-Padilla R, et al. Increased risk of exacerbation and hospitalization in subjects with an overlap phenotype: COPD-asthma. Chest. 2014;145(2):297-304.

33. Fu JJ, Gibson PG, Simpson JL, McDonald VM. Longitudinal changes in clinical outcomes in older patients with asthma, COPD and asthmaCOPD overlap syndrome. Respiration. 2014;87(1):63-74.

34. Fu JJ, McDonald VM, Gibson PG, Simpson JL. Systemic inflammation in older adults with asthma-COPD overlap syndrome. Allergy Asthma Immunol Res. 2014;6(4):316-324.

35. Harada T, Yamasaki A, Fukushima T, et al. Causes of death in patients with asthma and asthma-chronic obstructive pulmonary disease overlap syndrome. Int J Chron Obstruct Pulmon Dis. 2015;10:595-602.
36. Ding B, Enstone A. Asthma and chronic obstructive pulmonary disease overlap syndrome (ACOS): structured literature review and physician insights. Expert Rev Respir Med. 2016;10(3):363-371.

37. Janssens W, Liu Y, Liu D, et al. Quality and reproducibility of spirometry in COPD patients in a randomized trial (UPLIFT(R)). Respir Med. 2013;107(9):1409-1416.

38. Hasegawa K, Camargo CA Jr. Prevalence of blood eosinophilia in hospitalized patients with acute exacerbation of COPD. Respirology. 2016;21(4):761-764.

39. Alcazar-Navarrete B, Romero-Palacios PJ, Ruiz-Sancho A, Ruiz-Rodriguez O. Diagnostic performance of the measurement of nitric oxide in exhaled air in the diagnosis of COPD phenotypes. Nitric Oxide. 2016;54:67-72.

40. Chen FJ, Huang XY, Liu YL, Lin GP, Xie CM. Importance of fractional exhaled nitric oxide in the differentiation of asthma-COPD overlap syndrome, asthma, and COPD. Int J Chron Obstruct Pulmon Dis. 2016;11:2385-2390

41. Tamada T, Sugiura H, Takahashi T, et al. Biomarker-based detection of asthma-COPD overlap syndrome in COPD populations. Int J Chron Obstruct Pulmon Dis. 2015;10:2169-2176.

42. Lange P, Colak Y, Ingebrigtsen TS, Vestbo J, Marott JL. Longterm prognosis of asthma, chronic obstructive pulmonary disease, and asthma-chronic obstructive pulmonary disease overlap in the Copenhagen City Heart study: a prospective population-based analysis. Lancet Respir Med. 2016;4(6):454-462.

43. Postma DS, Rabe KF. The asthma-COPD overlap syndrome. $N$ Engl J Med. 2015;373(13):1241-1249.

44. D’Urzo A, Donohue JF, Kardos P, Miravitlles M, Price D. A reevaluation of the role of inhaled corticosteroids in the management of patients with chronic obstructive pulmonary disease. Expert Opin Pharmacother. 2015;16(12):1845-1860.

45. Gillissen A, Haidl P, Khlhaufl M, Kroegel K, Voshaar T, Gessner C. The pharmacological treatment of chronic obstructive pulmonary disease. Dtsch Arztebl Int. 2016;113(18):311-316.

46. Spencer S, Calverley PM, Burge PS, Jones PW. Impact of preventing exacerbations on deterioration of health status in COPD. Eur Respir $J$ 2004;23(5):698-702.

47. Global Initiative for Chronic Obstructive Lung Disease. GOLD Reports. Available from: http://goldcopd.org/gold-reports/. Accessed September 15, 2016.

48. Brusselle G, Price D, Gruffydd-Jones K, et al. The inevitable drift to triple therapy in COPD: an analysis of prescribing pathways in the UK. Int J Chron Obstruct Pulmon Dis. 2015;10:2207-2217.

49. Liesker JJ, Bathoorn E, Postma DS, Vonk JM, Timens W, Kerstjens HA. Sputum inflammation predicts exacerbations after cessation of inhaled corticosteroids in COPD. Respir Med. 2011;105(12):1853-1860.

50. Watz H, Tetzlaff K, Wouters EF, et al. Blood eosinophil count and exacerbations in severe chronic obstructive pulmonary disease after withdrawal of inhaled corticosteroids: a post-hoc analysis of the WISDOM trial. Lancet Respir Med. 2016;4(5):390-398. 


\section{Supplementary materials}

Table SI Major criteria for diagnosing ACOS

\begin{tabular}{ll}
\hline Criteria & $\mathbf{n}(\%)$ \\
\hline Reversibility in lung function and/or airway obstruction & $67(77)$ \\
History/diagnosis of asthma & $39(45)$ \\
Allergy/atopy & $38(44)$ \\
Smoking & $36(41)$ \\
Persistent airway obstruction & $22(25)$ \\
Eosinophilia/increased FeNO & $21(24)$ \\
COPD & $15(17)$ \\
Age & $19(22)$ \\
Exacerbations & $13(15)$ \\
Obstructive lung function & $11(13)$ \\
Variability in lung function and/or airway obstruction & $9(10)$ \\
Response to corticosteroids & $8(9)$ \\
Airway hyperresponsiveness & $7(8)$ \\
Lung diffusion & $5(6)$ \\
Variability in symptoms & $5(6)$ \\
Others & $38(44)$ \\
\hline Notes: &
\end{tabular}

Notes: aOthers: bronchospasm (4), cough (1), dyspnea (2), wheezing (2), chronic bronchitis (2), chronicity (1), response to therapy (2), clinic (4), lung function (I), impairment of bronchioles (I), emphysema (4), efficacy (I), availability (I), performance (I), spirometry (3), nocturnal symptoms (I), rhinitis (4), progressive (I), COPD in light smokers (I), and quality of life (I).

Abbreviations: ACOS, asthma-COPD overlap syndrome; $n$ (\%), number (percentage) of pulmonologists (total: 87); FeNO, fractional exhaled nitric oxide.

Table S2 Most important criteria to diagnose a COPD patient as ACOS patient

\begin{tabular}{lll}
\hline Items ( $\mathbf{n}=\mathbf{I 5})$ & Number of answers (N=26I) & \% of pulmonologists \\
\hline Degrees of response to bronchodilator testing during spirometry & 43 & 49.43 \\
Degree of variability in airway obstruction, tested by spirometry at different time points & 43 & 49.43 \\
Diagnosed with asthma before the age of 40 years & 40 & 45.98 \\
Personal or family history of atopy/(or, more specifically, IgE sensitivity to one or more & 39 & 44.83 \\
airborne allergens) & & 43.68 \\
Elevated eosinophil levels in blood or sputum, or increased FeNO & 38 & 22.99 \\
Symptoms variability & 20 & 22.99 \\
Former smoker/active smoker & 20 & 6.90 \\
Wheezing & 6 & 3.45 \\
Age & 3 & 3.45 \\
Nocturnal symptoms & 3 & 2.30 \\
Emphysema on chest CT scan & 2 & 2.30 \\
Reduced lung diffusion & 2 & 1.15 \\
Chronic cough & 1 & 1.15 \\
Increased lung volume (hyperinflation) & 1 & 0.00 \\
Sputum production & 0 &
\end{tabular}

Notes: aThe number and percentage of participants who identified the question two criterion specified as one of the three most important in the diagnosis of ACOS in a COPD patient. Dark and light gray underline, respectively, indicate major and minor criteria included in the ACOS diagnosis guidance.

Abbreviations: ACOS, asthma-COPD overlap syndrome; IgE, immunoglobulin E; FeNO, fractional exhaled nitric oxide; CT, computed tomography; N, total number of answers. 
Table S3 Most important criteria to diagnose an asthma patient as ACOS patient

\begin{tabular}{lll}
\hline Items ( $\mathbf{n}=\mathbf{I}$ ) & Number of answers $\mathbf{( N = 2 6 I ) ^ { \mathbf { a } }}$ & \% of pulmonologists $^{\mathbf{a}}$ \\
\hline Persistence over time of an obstructive disorder (no normalization) & $6 \mathrm{I}$ & $70.1 \mathrm{I}$ \\
Former smoker/active smoker & 57 & 65.52 \\
Emphysema on chest CT scan & 39 & 44.83 \\
Reduced lung diffusion & 28 & 32.18 \\
Degree of response to bronchodilators during spirometry & 18 & 20.69 \\
Degree of variability in airway obstruction, tested by spirometry & 16 & 18.39 \\
at different time points & & 10.34 \\
Age & 9 & 9.20 \\
Sputum production & 8 & 8.05 \\
Elevated blood or sputum eosinophils or increased FeNO & 7 & 5.75 \\
Increased lung volume (hyperinflation) & 5 & 4.60 \\
Symptoms variability & 4 & 4.60 \\
Chronic cough & 4 & 3.45 \\
Personal or family history of atopy & 3 & 2.30 \\
Nocturnal symptoms & 2 & 0.00 \\
Wheezing & 0 &
\end{tabular}

Notes: ${ }^{a}$ The number and percentage of participants who identified the question three criterion specified as one of the three most important in the diagnosis of ACOS in an asthma patient. Dark gray indicates the major criteria included in the ACOS diagnosis guidance, light gray the minor criteria.

Abbreviations: ACOS, asthma-COPD overlap syndrome; CT, computed tomography; FeNO, fractional exhaled nitric oxide; N, total number of answers.

\section{Publish your work in this journal}

The International Journal of COPD is an international, peer-reviewed journal of therapeutics and pharmacology focusing on concise rapid reporting of clinical studies and reviews in COPD. Special focus is given to the pathophysiological processes underlying the disease, intervention programs, patient focused education, and self management protocols.

\section{Dovepress}

This journal is indexed on PubMed Central, MedLine and CAS. The manuscript management system is completely online and includes a very quick and fair peer-review system, which is all easy to use. Visit $\mathrm{http}: / / \mathrm{www}$. dovepress.com/testimonials.php to read real quotes from published authors.

Submit your manuscript here: http://www.dovepress.com/international-journal-of-chronic-obstructive-pulmonary-disease-journal 